\title{
Multiparametric MRI scans could be a useful adjunct for active surveillance in prostate cancer
}

$\mathrm{M}$

ultiparametric MRI (mpMRI)a noninvasive technique that includes both anatomical and functional sequences-has been shown to be useful for prostate cancer detection and staging. However, the technique is not included in clinical algorithms. Now, two new publications provide further evidence that mpMRI has a role in prostate cancer management.

The first study, published in Radiology, investigated whether mpMRI could be used to identify patients who would be good candidates for active surveillance (AS) and compared the mpMRI stratification with currently used scoring systems, including the D'Amico, Epstein and Cancer of the Prostate Risk Assessment (CAPRA) scores at subsequent prostatectomy. AS offers the opportunity for men with low-volume, low-risk cancer to avoid unnecessary treatment-and its associated adverse effects-but is hampered by concerns that some patients would have been better served with surgery, as random biopsies are prone to inaccuracy. "Random biopsies sample only a small percentage of prostate volume and can miss or underestimate tumours," corresponding author Peter Choyke told Nature Reviews Urology. "This results in a high rate of upgrading at surgery and leads to uncertainty over the reliability of biopsy results." These inaccuracies are reflected in the aforementioned scores, which rely partly on biopsy results for stratification.

133 patients with a mean serum PSA level of $6.73 \mathrm{ng} / \mathrm{ml}$ underwent mpMRI before prostatectomy. After surgery, patients were retrospectively classified as to whether they would have been candidiates for AS according to mpMRI, Epstein, CAPRA, or D'Amico score. 14 patients were deemed to be appropriate for AS; sensitivity, positive predictive value (PPV) and overall accuracy of the D'Amico score for predicting AS candidates were $93 \%, 25 \%$, and $70 \%$, respectively.
Sensitivity, PPV and overall accuracy of the Epstein system were $64 \%, 45 \%$ and $88 \%$, respectively, and for the CAPRA score, $93 \%, 20 \%$ and $59 \%$, respectively. By comparison, sensitivity, PPV and overall accuracy of mpMRI for predicting patients suitable for AS were 93\%, 57\% and $92 \%$, respectively. 40 of 133 patients were misclassified using the D'Amico score, but on incorporation of mpMRI, 34 of these would have been correctly classified. Similar results were observed when mpMRI was incorporated into the other scoring systems, significantly improving accuracy. "The results showed that mpMRI was superior in sensitivity, PPV and overall accuracy to any of the three clinical scoring systems. When incorporated into those scoring systems, mpMRI produced even better results," concludes Choyke.

Furthermore, mpMRI can also be used to direct targeted biopsies, potentially improving scoring systems already in use and reducing the limitations associated with random biopsies. "mpMRI has been shown to be an accurate method of localizing and staging prostate cancer," explained first author Baris Turkbey.

"Recently, its utility in guiding prostate biopsies has also been reported. We report the utility of mpMRI in correctly stratifying patients with prostate cancer for AS or active treatment. We believe that incorporating mpMRI into existing nomograms will improve this stratification."

In a second recent publication, Mullins et al. investigated the utility of mpMRI for detecting index lesions in men already enrolled in an AS programme. 50 men under AS who were referred for prostate MRI had their images assessed by a radiologist. The presence of index lesions on MRI (MRI-index) — defined as a single suspicious lesion $\geq 10 \mathrm{~mm}$ in diameter or $>2$ lesions in a prostate sextant-was assessed, and clinical and pathological features compared between men with and without MRI-index. Pathology-identified index lesions (path-index) were defined as cancer present in a single prostate sextant on two biopsy sessions. mpMRI demonstrated a specificity of $97.4 \%$ and negative predictive value of $89.7 \%$ for detecting path-index lesions. Negative MRI correlated with a lack of path-index lesions and could, therefore, provide a noninvasive method of selecting and monitoring patients for AS.

Together these papers provide compelling evidence that mpMRI should be added to the clinical algorithms for AS. As Choyke concludes: "the use of mpMRI may enable more men to be safely managed with AS, while reassuring physicians that their patients have not missed the 'window of opportunity' for curative treatment."

\section{Annette Fenner}
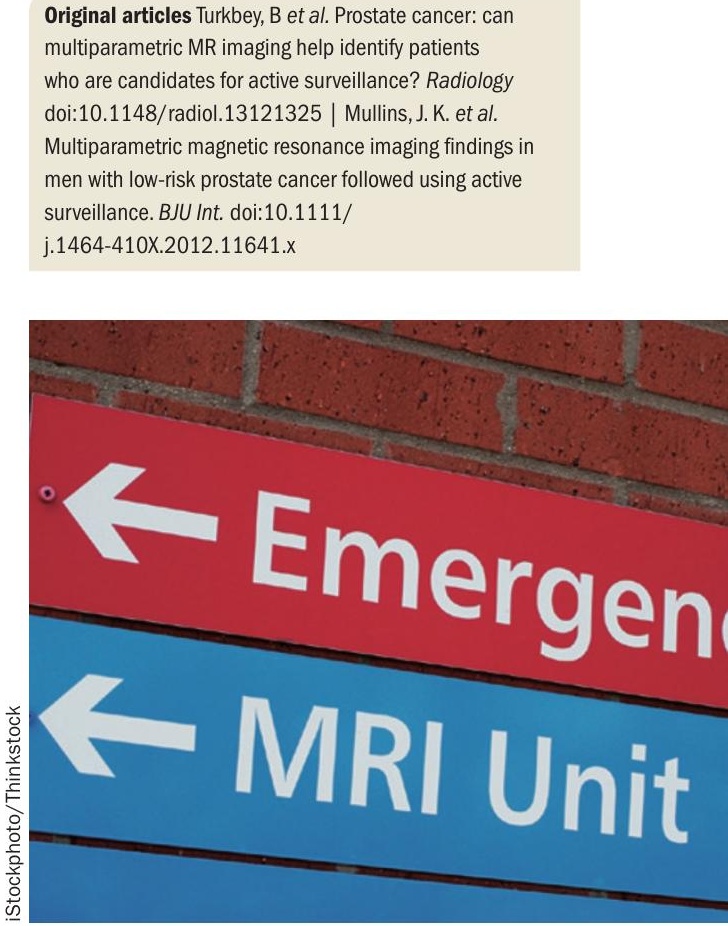